\title{
Singular integral equation involving a multivariable analog of Mittag-Leffler function
}

\author{
Sebastien Gaboury ${ }^{\text {* }}$ and Mehmet Ali Özarslan²
}

\section{"Correspondence:}

s1gabour@uqac.ca

${ }^{1}$ Department of Mathematics and

Computer Science, University of

Quebec at Chicoutimi, Quebec, G7H 2B1, Canada

Full list of author information is

available at the end of the article

\begin{abstract}
Motivated by the recent work of the second author (Özarslan in Appl. Math. Comput. 229:350-358, 2014), we present, in this paper, some fractional calculus formulas for a mild generalization of the multivariable Mittag-Leffler function, a Schläfli's type contour integral representation, some multilinear and mixed multilateral generating functions; and, finally, we consider a singular integral equation with the function $E_{\left(\rho_{r}\right) \lambda}^{\left(\gamma_{r},(1)\right.}\left(x_{1}, \ldots, x_{r}\right)$ in the kernel and we provide its solution.

MSC: $26 \mathrm{~A} 33 ; 33 \mathrm{E} 12$
\end{abstract}

Keywords: fractional integrals and derivatives; Mittag-Leffler function; contour integral representation; generating functions; singular integral equation; Laplace transform

\section{Introduction}

The celebrated Mittag-Leffler function [1,2] is defined by

$$
\begin{aligned}
& E_{\alpha}(z)=\sum_{k=0}^{\infty} \frac{z^{k}}{\Gamma(\alpha k+1)} \\
& (\alpha \in \mathbb{C} ; \Re(\alpha)>0 ; z \in \mathbb{C}),
\end{aligned}
$$

where $\mathbb{C}$ denotes the set of complex numbers.

The Mittag-Leffler function arises naturally in the solution of fractional integral equations [3]. A generalization of the Mittag-Leffler function $E_{\alpha}(z)$ has been investigated by Wiman [4]. He studied the following function:

$$
\begin{aligned}
& E_{\alpha, \beta}(z)=\sum_{k=0}^{\infty} \frac{z^{k}}{\Gamma(\alpha k+\beta)} \\
& (\alpha, \beta \in \mathbb{C} ; \Re(\alpha)>0 ; \Re(\beta)>0 ; z \in \mathbb{C}) .
\end{aligned}
$$

Other generalizations of the Mittag-Leffler functions were given in $[5,6]$. Let us recall the one given by Srivastava and Tomovski [6]:

@2014 Gaboury and Özarslan; licensee Springer. This is an Open Access article distributed under the terms of the Creative Commons Attribution License (http://creativecommons.org/licenses/by/2.0), which permits unrestricted use, distribution, and reproduction in any medium, provided the original work is properly cited. 


$$
\begin{aligned}
& E_{\alpha, \beta}^{\gamma, K}(z)=\sum_{k=0}^{\infty} \frac{(\gamma)_{K n}}{\Gamma(\alpha k+\beta)} \frac{z^{k}}{k !} \\
& (\alpha, \beta, \gamma \in \mathbb{C} ; \Re(\alpha)>\max \{0, \Re(K)-1\} ; \Re(K)>0 ; \Re(\beta)>0 ; z \in \mathbb{C}),
\end{aligned}
$$

where $(\lambda)_{\kappa}$ denotes the Pochhammer symbol defined, in terms of the Gamma function, by

$$
(\lambda)_{\kappa}:=\frac{\Gamma(\lambda+\kappa)}{\Gamma(\lambda)}= \begin{cases}\lambda(\lambda+1) \cdots(\lambda+n-1) & (\kappa=n \in \mathbb{N} ; \lambda \in \mathbb{C}), \\ 1 & (\kappa=0 ; \lambda \in \mathbb{C} \backslash\{0\}),\end{cases}
$$

where $\mathbb{N}$ denotes the set of positive integers.

Multivariable analog of the Mittag-Leffler function has been introduced and investigated by Saxena et al. [7, p.536, Eq. (1.14)] in the following form:

$$
\begin{aligned}
& E_{\left(\rho_{r}\right), \lambda}^{\left(\gamma_{r}\right)}\left(z_{1}, \ldots, z_{r}\right)=E_{\left(\rho_{1}, \ldots, \rho_{r}\right), \lambda}^{\left(\gamma_{1}, \ldots, \gamma_{r}\right)}\left(z_{1}, \ldots, z_{r}\right) \\
&=\sum_{k_{1}, \ldots, k_{r}=0}^{\infty} \frac{\left(\gamma_{1}\right)_{k_{1}} \cdots\left(\gamma_{r}\right)_{k_{r}}}{\Gamma\left(k_{1} \rho_{1}+\cdots+k_{r} \rho_{r}+\lambda\right)} \frac{z_{1}^{k_{1}} \cdots z_{r}^{k_{r}}}{k_{1} ! \cdots k_{r} !} \\
&\left(\lambda, z_{j}, \gamma_{j}, \rho_{j} \in \mathbb{C} ; \Re\left(\rho_{j}\right)>0 ; j=1,2, \ldots, r\right) .
\end{aligned}
$$

This function is, in fact, a special case of the generalized Lauricella series in several variables, introduced by Srivastava and Daoust [8] and Srivastava and Karlsson [9].

A mild generalization of the multivariable analog of the Mittag-Leffler function, which will play an important role in this paper, has been given by Saxena et al. [7, p.547, Eq. (7.1)]:

$$
\begin{aligned}
& E_{\left(\rho_{r}\right), \lambda}^{\left(\gamma_{r}\right),\left(l_{r}\right)}\left(z_{1}, \ldots, z_{r}\right)=\sum_{k_{1}, \ldots, k_{r}=0}^{\infty} \frac{\left(\gamma_{1}\right)_{k_{1} l_{1}} \cdots\left(\gamma_{r}\right)_{k_{r} l_{r}}}{\Gamma\left(k_{1} \rho_{1}+\cdots+k_{r} \rho_{r}+\lambda\right)} \frac{z_{1}^{k_{1}} \cdots z_{r}^{k_{r}}}{k_{1} ! \cdots k_{r} !} \\
& \left(\lambda \in \mathbb{C} \backslash \mathbb{Z}_{0}^{-} ; \gamma_{j}, \rho_{j}, l_{j} \in \mathbb{C} ; \Re\left(\rho_{j}\right)>0 ; \Re\left(l_{j}\right)>0 ; j=1,2, \ldots, r\right) .
\end{aligned}
$$

Recently, the second author in [10] introduced a class of polynomials suggested by the multivariate Laguerre polynomials in the following form:

$$
\begin{aligned}
& Z_{n_{1}, \ldots, n_{r}}^{(\alpha)}\left(x_{1}, \ldots, x_{r} ; \rho_{1}, \ldots, \rho_{r}\right) \\
& \quad=\frac{\Gamma\left(\rho_{1} n_{1}+\cdots+\rho_{r} n_{r}+\alpha+1\right)}{n_{1} ! \cdots n_{r} !} \sum_{k_{1}, \ldots, k_{r}}^{n_{1}, \ldots, n_{r}} \frac{\left(-n_{1}\right)_{k_{1}} \cdots\left(-n_{r}\right)_{k_{r}} x_{1}^{\rho_{1} k_{1}} \cdots x_{r}^{\rho_{r} k_{r}}}{\Gamma\left(\rho_{1} k_{1}+\cdots+\rho_{r} k_{r}+\alpha+1\right) k_{1} ! \cdots k_{r} !} \\
& \left(\alpha, \rho_{1}, \ldots, \rho_{r} \in \mathbb{C} ; \Re\left(\rho_{j}\right)>0(j=1,2, \ldots, r)\right) .
\end{aligned}
$$

It is easy to see that the following relation between the class of polynomials given by (1.7) and the generalized multivariable Mittag-Leffler function (1.6) exists:

$$
\begin{aligned}
& Z_{n_{1}, \ldots, n_{r}}^{(\alpha)}\left(x_{1}, \ldots, x_{r} ; \rho_{1}, \ldots, \rho_{r}\right) \\
& \quad=\frac{\Gamma\left(\rho_{1} n_{1}+\cdots+\rho_{r} n_{r}+\alpha+1\right)}{n_{1} ! \cdots n_{r} !} E_{\left(\rho_{1}, \ldots, \rho_{r}\right), \alpha+1}^{\left(-n_{1}, \ldots,-n_{r}\right),(1, \ldots, 1)}\left(x_{1}^{\rho_{1}}, \ldots, x_{r}^{\rho_{r}}\right) .
\end{aligned}
$$


Note that by further specializing the several parameters involved, we can obtain many well-known classes of polynomials such as the Laguerre polynomials of $r$ variables defined by Erdélyi [11] and the Konhauser polynomials [12].

Another interesting generalization of the polynomials $Z_{n_{1}, \ldots, n_{r}}^{(\alpha)}\left(x_{1}, \ldots, x_{r} ; \rho_{1}, \ldots, \rho_{r}\right)$ is given by

$$
\begin{aligned}
& Z_{n_{1}, \ldots, n_{r}}^{\left(\alpha ; N_{1}, \ldots, N_{r}\right)}\left(x_{1}, \ldots, x_{r} ; \rho_{1}, \ldots, \rho_{r}\right) \\
& \quad=\frac{\Gamma\left(\rho_{1} n_{1}+\cdots+\rho_{r} n_{r}+\alpha+1\right)}{n_{1} ! \cdots n_{r} !} \sum_{k_{1}, \ldots, k_{r}=0}^{\left[\frac{n_{1}}{N_{1}}\right], \ldots,\left[\frac{n_{r}}{N_{r}}\right]} \frac{\left(-n_{1}\right)_{N_{1} k_{1}} \cdots\left(-n_{r}\right)_{N_{r} k_{r}} x_{1}^{\rho_{1} k_{1}} \cdots x_{r}^{\rho_{r} k_{r}}}{\Gamma\left(\rho_{1} k_{1}+\cdots+\rho_{r} k_{r}+\alpha+1\right) k_{1} ! \cdots k_{r} !} \\
& \left(\alpha, \rho_{1}, \ldots, \rho_{r} \in \mathbb{C}, \Re\left(\rho_{i}\right)>0, N_{i} \in \mathbb{N}(i=1, \ldots, r)\right) .
\end{aligned}
$$

Obviously, setting $N_{i}=1(i=1, \ldots, r)$ leads to $(1.8)$.

In this paper, we obtain a Schläfli's type contour integral representation for the multivariable polynomials given in (1.9). Next, we give some multilinear and mixed multilateral generating functions. We also recall the fractional order integral of the generalized multivariable Mittag-Leffler function. Finally, we consider a singular integral equation with $E_{\left(\rho_{r}\right), \lambda}^{\left(\gamma_{r}\right),(1)}\left(x_{1}, \ldots, x_{r}\right)$ in the kernel and we give its solution. Throughout this paper, the variables $x_{1}, \ldots, x_{r}$ are assumed to be real variables.

\section{Schläfli's type contour integral representation of $Z_{n_{1}, \ldots, n_{r}}^{\left(\alpha, N_{1}, \ldots, N_{r}\right)}\left(x_{1}, \ldots, x_{r}\right.$;}

\section{$\left.\rho_{1}, \ldots, \rho_{r}\right)$}

Let us define the following polynomials set:

$$
\begin{aligned}
& P_{n_{1}, \ldots, n_{r}}^{\left(N_{1}, \ldots, N_{r}\right)}\left(x_{1}, \ldots, x_{r} ; \rho_{1}, \ldots, \rho_{r}\right) \\
& \quad:=\sum_{k_{1}, \ldots, k_{r}=0}^{\left\lceil\frac{n_{1}}{N_{1}}\right] \ldots,\left[\frac{n_{r}}{N_{r}}\right]}\left(-n_{1}\right)_{N_{1} k_{1}} \cdots\left(-n_{r}\right)_{N_{r} k_{r}} \frac{x_{1}^{\rho_{1} k_{1}} \cdots x_{r}^{\rho_{r} k_{r}}}{k_{1} ! \cdots k_{r} !} \\
& \left(\rho_{j} \in \mathbb{C} ; \Re\left(\rho_{j}\right)>0 ; N_{j} \in \mathbb{N}(j=1, \ldots, r)\right) .
\end{aligned}
$$

The Schläfli's type contour integral representation of $Z_{n_{1}, \ldots, n_{r}}^{\left(\alpha, \ldots, N_{r}\right)}\left(x_{1}, \ldots, x_{r} ; \rho_{1}, \ldots, \rho_{r}\right)$ in terms of $P_{n_{1}, \ldots, n_{r}}^{\left(N_{1}, \ldots, N_{r}\right)}\left(x_{1}, \ldots, x_{r} ; \rho_{1}, \ldots, \rho_{r}\right)$ is given in the next theorem.

Theorem 2.1 Let $\alpha, \rho_{j} \in \mathbb{C}$ with $\Re\left(\rho_{j}\right)>0(j=1, \ldots, r)$ and let $N_{j} \in \mathbb{N}(j=1, \ldots, r)$. Then the following integral representation holds true:

$$
\begin{aligned}
Z_{n_{1}, \ldots, n_{r}}^{\left(\alpha ; N_{1}, \ldots, N_{r}\right)}\left(x_{1}, \ldots, x_{r} ; \rho_{1}, \ldots, \rho_{r}\right) \\
=\frac{\Gamma\left(\rho_{1} n_{1}+\cdots+\rho_{r} n_{r}+\alpha+1\right)}{n_{1} ! \cdots n_{r} !} \frac{1}{2 \pi i} \\
\quad \times \int_{-\infty}^{(0+)} P_{n_{1}, \ldots, n_{r}}^{\left(N_{1}, \ldots, N_{r}\right)}\left(\frac{x_{1}}{t}, \ldots, \frac{x_{r}}{t} ; \rho_{1}, \ldots, \rho_{r}\right) t^{-\alpha-1} \mathrm{e}^{t} \mathrm{~d} t .
\end{aligned}
$$


Proof We have

$$
\begin{aligned}
& \frac{1}{2 \pi i} \int_{-\infty}^{(0+)} P_{n_{1}, \ldots, n_{r}}^{\left(N_{1}, \ldots N_{r}\right)}\left(\frac{x_{1}}{t}, \ldots, \frac{x_{r}}{t} ; \rho_{1}, \ldots, \rho_{r}\right) t^{-\alpha-1} \mathrm{e}^{t} \mathrm{~d} t \\
& \quad=\frac{1}{2 \pi i} \int_{-\infty}^{(0+)} \sum_{k_{1}, \ldots, k_{r}=0}^{\left[\frac{n_{1}}{N_{1}}\right], \ldots,\left[\frac{n_{r}}{\left.N_{r}\right]}\right.} \frac{\left(-n_{1}\right)_{N_{1} k_{1}} \cdots\left(-n_{r}\right)_{N_{r} k_{r}}}{k_{1} ! \cdots k_{r} !} x_{1}^{\rho_{1} k_{1}} \cdots x_{r}^{\rho_{r} k_{r}} \frac{\mathrm{e}^{t}}{t^{\rho_{1} k_{1}+\cdots+\rho_{r} k_{r}+\alpha+1}} \mathrm{~d} t .
\end{aligned}
$$

With the help of Hankel's formula [13]

$$
\frac{1}{\Gamma(z)}=\frac{1}{2 \pi i} \int_{-\infty}^{(0+)} t^{-z} \mathrm{e}^{t} \mathrm{~d} t
$$

we find from (2.3) and (2.4) the result asserted by Theorem 2.1.

\section{Multilinear and multilateral generating functions}

We begin this section by proving a linear generating function for the polynomials $Z_{n_{1}, \ldots, n_{j}}^{\left(\alpha ; N_{1}, \ldots, N_{j}\right)}\left(x_{1}, \ldots, x_{j} ; \rho_{1}, \ldots, \rho_{j}\right)$ by means of the mild generalization of the multivariate analog of Mittag-Leffler functions.

Theorem 3.1 We have

$$
\begin{aligned}
& \sum_{n_{1}, \ldots, n_{j}=0}^{\infty} \frac{\left(\gamma_{1}\right)_{n_{1}} \cdots\left(\gamma_{j}\right)_{n_{j}} Z_{n_{1}, \ldots, n_{j}}^{\left(\alpha ; N_{1}, \ldots, N_{j}\right)}\left(x_{1}, \ldots, x_{j} ; \rho_{1}, \ldots, \rho_{j}\right)}{\Gamma\left(\rho_{1} n_{1}+\cdots+\rho_{j} n_{j}+\alpha+1\right)} t_{1}^{n_{1}} \cdots t_{j}^{n_{j}} \\
& \quad=\prod_{i=1}^{j}\left(1-t_{i}\right)^{-\gamma_{i}} E_{\rho_{1}, \ldots, \rho_{j}, \alpha+1}^{\left(\gamma_{j}\right),\left(N_{j}\right)}\left(\frac{x_{1}^{\rho_{1}}\left(-t_{1}\right)^{N_{1}}}{\left(1-t_{1}\right)^{N_{1}}}, \ldots, \frac{x_{j}^{\rho_{j}}\left(-t_{j}\right)^{N_{j}}}{\left(1-t_{j}\right)^{N_{j}}}\right),
\end{aligned}
$$

where $\left|t_{i}\right|<1(i=1, \ldots, j)$.

Proof Direct calculations yield

$$
\begin{aligned}
& \sum_{n_{1}, \ldots, n_{j}=0}^{\infty} \frac{\left(\gamma_{1}\right)_{n_{1}} \cdots\left(\gamma_{j}\right)_{n_{j}} Z_{n_{1}, \ldots, n_{j}}^{\left(\alpha ; N_{1}, \ldots, N_{j}\right)}\left(x_{1}, \ldots, x_{j} ; \rho_{1}, \ldots, \rho_{j}\right)}{\Gamma\left(\rho_{1} n_{1}+\cdots+\rho_{j} n_{j}+\alpha+1\right)} t_{1}^{n_{1}} \cdots t_{j}^{n_{j}} \\
& =\sum_{n_{1}, \ldots, n_{j}=0}^{\infty} \sum_{k_{1}, \ldots, k_{j}=0}^{\left[\frac{n_{1}}{N_{1}}\right], \ldots,\left[\frac{n_{j}}{N_{j}}\right]} \frac{(-1)^{N_{1} k_{1}+\cdots+N_{j} k_{j}}\left(\gamma_{1}\right)_{n_{1}} \cdots\left(\gamma_{j}\right)_{n_{j}} x_{1}^{\rho_{1} k_{1}} \cdots x_{j}^{\rho_{j} k_{j}} t_{1}^{n_{1}} \cdots t_{j}^{n_{j}}}{\Gamma\left(\rho_{1} k_{1}+\cdots+\rho_{j} k_{j}+\alpha+1\right)\left(n_{1}-N_{1} k_{1}\right) ! \cdots\left(n_{j}-N_{k} k_{j}\right) ! k_{1} ! \cdots k_{j} !} \\
& =\sum_{n_{1}, \ldots, n_{j}=0}^{\infty} \sum_{k_{1}, \ldots, k_{j}=0}^{\infty} \frac{(-1)^{N_{1} k_{1}+\cdots+N_{j} k_{j}}\left(\gamma_{1}\right)_{n_{1}+N_{1} k_{1}} \cdots\left(\gamma_{j}\right)_{n_{j}+N_{j} k_{j}} x_{1}^{\rho_{1} k_{1}} \cdots x_{j}^{\rho_{j} k_{j}} t_{1}^{n_{1}+N_{1} k_{1}} \cdots t_{j}^{n_{j}+N_{j} k_{j}}}{\Gamma\left(\rho_{1} k_{1}+\cdots+\rho_{j} k_{j}+\alpha+1\right) n_{1} ! \cdots n_{j} ! k_{1} ! \cdots k_{j} !} \\
& =\sum_{k_{1}, \ldots, k_{j}=0}^{\infty} \frac{\left(\gamma_{1}\right)_{N_{1} k_{1}} \cdots\left(\gamma_{j}\right)_{N_{j} k_{j}}\left(x_{1}^{\rho_{1}}\left(-t_{1}\right)^{N_{1}}\right)^{k_{1}} \cdots\left(x_{j}^{\rho_{j}}\left(-t_{j}\right)^{N_{j}}\right)^{k_{j}}}{\Gamma\left(\rho_{1} k_{1}+\cdots+\rho_{j} k_{j}+\alpha+1\right) k_{1} ! \cdots k_{j} !} \\
& \times \sum_{n_{1}, \ldots, n_{j}=0}^{\infty} \frac{\left(\gamma_{1}+N_{1} k_{1}\right)_{n_{1}} \cdots\left(\gamma_{j}+N_{j} k_{j}\right)_{n_{j}}}{n_{1} ! \cdots n_{j} !} t_{1}^{n_{1}} \cdots t_{j}^{n_{j}} \\
& =\prod_{i=1}^{j}\left(1-t_{i}\right)^{-\gamma_{i}} E_{\rho_{1}, \ldots, \rho_{j}, \alpha+1}^{\left(\gamma_{j}\right),\left(N_{j}\right)}\left(\frac{x_{1}^{\rho_{1}}\left(-t_{1}\right)^{N_{1}}}{\left(1-t_{1}\right)^{N_{1}}}, \ldots, \frac{x_{j}^{\rho_{j}}\left(-t_{j}\right)^{N_{j}}}{\left(1-t_{j}\right)^{N_{j}}}\right),
\end{aligned}
$$


where we have interchanged the order of summations which is guaranteed because of the uniform convergence of the series under the conditions $\left|t_{i}\right|<1(i=1, \ldots, j)$.

Now let $(\gamma):=\left(\gamma_{1}, \ldots \gamma_{j}\right),(\lambda):=\left(\lambda_{1}, \ldots, \lambda_{j}\right),(\eta):=\left(\eta_{1}, \ldots, \eta_{j}\right),(\psi):=\left(\psi_{1}, \ldots, \psi_{j}\right),(\rho):=$ $\left(\rho_{1}, \ldots, \rho_{j}\right),(N):=\left(N_{1}, \ldots, N_{j}\right)$ be complex $j$-tuples. By making use of the above theorem we have the following.

Theorem 3.2 Corresponding to an identically non-vanishing function $\Omega_{(\eta)}\left(\xi_{1}, \ldots, \xi_{s}\right)$ of complex variables $\xi_{1}, \ldots, \xi_{s}(s \in \mathbb{N})$, let

$$
\begin{aligned}
& \Lambda_{(\eta),(\psi)}\left(\xi_{1}, \ldots, \xi_{s} ; \varsigma_{1}, \ldots, \varsigma_{j}\right) \\
& \quad:=\sum_{k_{1}, \ldots, k_{j}=0}^{\infty} a_{k_{1}, \ldots, k_{j}} \Omega_{\eta_{1}+\psi_{1} k_{1}, \ldots, \eta_{j}+\psi_{j} k_{j}}\left(\xi_{1}, \ldots, \xi_{s}\right) \varsigma_{1}^{k_{1}} \cdots \varsigma_{j}^{k_{j}} \quad\left(a_{k_{1}, \ldots, k_{j}} \neq 0\right) .
\end{aligned}
$$

Suppose also that

$$
\begin{aligned}
& \Theta_{n_{1}, \ldots, n_{j} ; q_{1}, \ldots, q_{j}}^{(\gamma),(\lambda),(\lambda),(\psi), \alpha((N)}\left(\xi_{1}, \ldots, \xi_{s} ; x_{1}, \ldots, x_{j} ;(\rho) ; \varsigma_{1}, \ldots, \varsigma_{j}\right) \\
& {\left[\frac{n_{1}}{q_{1}}\right], \ldots\left[\frac{n_{j}}{k_{j}}\right]} \\
& =\sum_{k_{1}, \ldots, k_{j}=0} a_{k_{1}, \ldots, k_{j}} \Omega_{\eta_{1}+\psi_{1} k_{1}, \ldots, \eta_{j}+\psi_{j} k_{j}}\left(\xi_{1}, \ldots, \xi_{s}\right) \\
& \times \frac{\left(\gamma_{1}+\lambda_{1} k_{1}\right)_{n_{1}-q_{1} k_{1}} \cdots\left(\gamma_{j}+\lambda_{j} k_{j}\right)_{n_{j}-q_{j} k_{j}} Z_{n_{1}-q_{1} k_{1}, \ldots, n_{j}-q_{j} k_{j}}^{\left(\alpha ; N_{1}, \ldots, N_{j}\right)}\left(x_{1}, \ldots, x_{j} ; \rho_{1}, \ldots, \rho_{j}\right)}{\Gamma\left(\rho_{1}\left(n_{1}-q_{1} k_{1}\right)+\cdots+\rho_{j}\left(n_{j}-q_{j} k_{j}\right)+\alpha+1\right)} \\
& \times \varsigma_{1}^{k_{1}} \cdots \varsigma_{j}^{k_{j}} \quad\left(q_{1}, \ldots, q_{j} \in \mathbb{N}\right) .
\end{aligned}
$$

Then

$$
\begin{aligned}
& \sum_{n_{1}, \ldots, n_{j}=0}^{\infty} \Theta_{n_{1}, \ldots, n_{j} ; q_{1}, \ldots, q_{j}}^{(\gamma),(,),(N)}\left(\xi_{1}, \ldots, \xi_{s} ; x_{1}, \ldots, x_{j} ;(\rho) ; \frac{\varsigma_{1}}{t_{1}^{q_{1}}}, \ldots, \frac{\varsigma_{j}}{t_{j}^{q_{j}}}\right) t_{1}^{n_{1}} \cdots t_{j}^{n_{j}} \\
& =\prod_{i=1}^{j}\left(1-t_{i}\right)^{-\gamma_{i}} \Lambda_{(\eta),(\psi)}\left(\xi_{1}, \ldots, \xi_{s} ; \frac{\varsigma_{1}}{\left(1-t_{1}\right)^{\lambda_{1}}}, \ldots, \frac{\varsigma_{j}}{\left(1-t_{j}\right)^{\lambda_{j}}}\right) \\
& \quad \times E_{\rho_{1}, \ldots, \rho_{j}, \alpha+1}^{\left(\gamma_{j},\left(N_{j}\right)\right.}\left(\frac{x_{1}^{\rho_{1}}\left(-t_{1}\right)^{N_{1}}}{\left(1-t_{1}\right)^{N_{1}}}, \ldots, \frac{x_{j}^{\rho_{j}}\left(-t_{j}\right)^{N_{j}}}{\left(1-t_{j}\right)^{N_{j}}}\right),
\end{aligned}
$$

provided that each member of $(3.3)$ exists and $\left|t_{i}\right|<1(i=1, \ldots, j)$.

Proof Following similar lines to [10], the proof is completed.

\section{Fractional integrals and derivatives}

In this section, we first recall the definitions of the Riemann-Liouville fractional integrals and derivatives. Next, we give the fractional integral and derivative of the generalized multivariable Mittag-Leffler function $E_{\left(\rho_{r}\right), \lambda}^{\left(\gamma_{r}\right),\left(l_{r}\right)}\left(x_{1}, \ldots, x_{r}\right)$ where $x_{j}$ are real variables for $j=1, \ldots, r$. 
Definition 4.1 Let $\Omega=[a, b]$ be a finite interval of the real axis. The Riemann-Liouville fractional integral of order $\alpha \in \mathbb{C}$ with $\Re(\alpha)>0$ is defined by

$$
{ }_{x} I_{a^{+}}^{\alpha}[f]=\frac{1}{\Gamma(\alpha)} \int_{a}^{x} \frac{f(t) d t}{(x-t)^{1-\alpha}} \quad(x>a) .
$$

It is well known $[14, \mathrm{p} .71]$ that

$$
{ }_{x} I_{0^{+}}^{\alpha}\left[x^{p}\right]=\frac{\Gamma(1+p)}{\Gamma(1+p+\alpha)} x^{p+\alpha} \quad(\Re(\alpha)>0 ; \Re(p)>-1) .
$$

Definition 4.2 Let $\Omega=[a, b]$ be a finite interval of the real axis. The Riemann-Liouville fractional derivative of order $\alpha \in \mathbb{C}$ with $\Re(\alpha) \geq 0$ is defined by

$$
{ }_{x} D_{a^{+}}^{\alpha}[f]=\left(\frac{d}{d x}\right)_{x}^{n} I_{a^{+}}^{n-\alpha}[f] \quad(n=[\Re(\alpha)]+1 ; x>a),
$$

where $[\Re(\alpha)]$ denotes the integral part of $\Re(\alpha)$.

Using (4.2), we see easily that

$$
{ }_{x} D_{0^{+}}^{\alpha}\left[x^{p}\right]=\frac{\Gamma(1+p)}{\Gamma(1+p-\alpha)} x^{p-\alpha} \quad(\Re(\alpha) \geq 0 ; \Re(p)>-1) .
$$

Now, let us give two fractional calculus formulas obtained by Jaimini and Gupta [15, p.145, Eqs. (1) and (2)] involving the generalized multivariable Mittag-Leffler function.

Theorem 4.3 Let $\alpha, \lambda, \rho_{j}, \gamma_{j}, l_{j}, \omega_{j} \in \mathbb{C}$ such that $\Re(\alpha)>0 ; \Re(\lambda)>0 ; \Re\left(\rho_{j}\right)>0 ; \Re\left(l_{j}\right)>0$ $(j=1, \ldots, r)$. Then the following fractional calculus formulas:

$$
{ }_{x} I_{0^{+}}^{\alpha}\left[x^{\lambda-1} E_{\left(\rho_{r}\right), \lambda}^{\left(\gamma_{r}\right),\left(l_{r}\right)}\left(\omega_{1} x^{\rho_{1}}, \ldots, \omega_{r} x^{\rho_{r}}\right)\right]=x^{\lambda+\alpha-1} E_{\left(\rho_{r}\right), \lambda+\alpha}^{\left(\gamma_{r}\right),\left(l_{r}\right)}\left(\omega_{1} x^{\rho_{1}}, \ldots, \omega_{r} x^{\rho_{r}}\right)
$$

and

$$
{ }_{x} D_{0^{+}}^{\alpha}\left[x^{\lambda-1} E_{\left(\rho_{r}\right), \lambda}^{\left(\gamma_{r}\right),\left(l_{r}\right)}\left(\omega_{1} x^{\rho_{1}}, \ldots, \omega_{r} x^{\rho_{r}}\right)\right]=x^{\lambda-\alpha-1} E_{\left(\rho_{r}\right), \lambda-\alpha}^{\left(\gamma_{r}\right),\left(l_{r}\right)}\left(\omega_{1} x^{\rho_{1}}, \ldots, \omega_{r} x^{\rho_{r}}\right)
$$

hold true.

Setting $\lambda=\lambda+1, l_{j}=\omega_{j}=1(j=1, \ldots, r)$, replacing $\gamma_{1}, \ldots, \gamma_{r}$, respectively, by $-n_{1}, \ldots,-n_{r}$, where $n_{j}(j=1, \ldots, r)$ are positive integers in (4.5) and (4.6), and making use of (1.8) yield the following special cases given by Özarslan [10, p.353, Theorem 6 and Theorem 8]:

$$
\begin{aligned}
{ }_{x} I_{0^{+}}^{\alpha} & {\left[x^{\lambda} Z_{n_{1}, \ldots, n_{r}}^{(\lambda)}\left(x, \ldots, x ; \rho_{1}, \ldots, \rho_{r}\right)\right] } \\
& =\frac{\Gamma\left(\rho_{1} n_{1}+\cdots+\rho_{r} n_{r}+\lambda+1\right)}{\Gamma\left(\rho_{1} n_{1}+\cdots+\rho_{r} n_{r}+\lambda+\alpha+1\right)} x^{\lambda+\alpha} Z_{n_{1}, \ldots, n_{r}}^{(\lambda+\alpha)}\left(x, \ldots, x ; \rho_{1}, \ldots, \rho_{r}\right)
\end{aligned}
$$

and

$$
\begin{aligned}
{ }_{x} D_{0^{+}}^{\alpha}\left[x^{\lambda} Z_{n_{1}, \ldots, n_{r}}^{(\lambda)}\left(x, \ldots, x ; \rho_{1}, \ldots, \rho_{r}\right)\right] \\
=\frac{\Gamma\left(\rho_{1} n_{1}+\cdots+\rho_{r} n_{r}+\lambda+1\right)}{\Gamma\left(\rho_{1} n_{1}+\cdots+\rho_{r} n_{r}+\lambda-\alpha+1\right)} x^{\lambda-\alpha} Z_{n_{1}, \ldots, n_{r}}^{(\lambda+\alpha}\left(x, \ldots, x ; \rho_{1}, \ldots, \rho_{r}\right) .
\end{aligned}
$$


Further special cases of (4.5) and (4.6) can be obtained by suitably specializing the coefficients involved. For instance, if we set $l_{j}=1(j=1, \ldots, r)$, then (4.5) and (4.6) reduce to two results obtained by Saxena et al. [7].

We end this section by giving a recurrence relation for the generalized multivariable Mittag-Leffler function $E_{\left(\rho_{r}\right), \lambda}^{\left(\gamma_{r}\right),\left(l_{r}\right)}\left(z_{1}, \ldots, z_{r}\right)$.

Theorem 4.4 Let $\lambda, \rho_{j}, \gamma_{j}, l_{j} \in \mathbb{C}$ such that $\Re(\lambda)>0$; $\Re\left(\rho_{j}\right)>0$; $\Re\left(l_{j}\right)>0(j=1, \ldots, r)$. Then the following recurrence relation holds true:

$$
E_{\left(\rho_{r}\right), \lambda}^{\left(\gamma_{r}\right),\left(l_{r}\right)}\left(z_{1}, \ldots, z_{r}\right)=\lambda E_{\left(\rho_{r}\right), \lambda+1}^{\left(\gamma_{r}\right),\left(l_{r}\right)}\left(z_{1}, \ldots, z_{r}\right)+\sum_{i=1}^{r} \rho_{i} z_{i} \frac{\partial}{\partial z_{i}} E_{\left(\rho_{r}\right), \lambda+1}^{\left(\gamma_{r}\right),\left(l_{r}\right)}\left(z_{1}, \ldots, z_{r}\right) .
$$

Proof From (1.6), we have

$$
\begin{aligned}
& \lambda E_{\left(\rho_{r}\right), \lambda+1}^{\left(\gamma_{r}\right),\left(l_{r}\right)}\left(z_{1}, \ldots, z_{r}\right)+\sum_{i=1}^{r} \rho_{i} z_{i} \frac{\partial}{\partial z_{i}} E_{\left(\rho_{r}\right), \lambda+1}^{\left(\gamma_{r}\right),\left(l_{r}\right)}\left(z_{1}, \ldots, z_{r}\right) \\
& \quad=\sum_{k_{1}, \ldots, k_{r}=0}^{\infty} \frac{\left(\gamma_{1}\right)_{k_{1} l_{1}} \cdots\left(\gamma_{r}\right)_{k_{r} l_{r}}}{\Gamma\left(k_{1} \rho_{1}+\cdots+k_{r} \rho_{r}+\lambda+1\right)}\left[\lambda+\rho_{1} k_{1}+\cdots+\rho_{r} k_{r}\right] \frac{z_{1}^{k_{1}} \cdots z_{r}^{k_{r}}}{k_{1} ! \cdots k_{r} !} \\
& \quad=E_{\left(\rho_{r}\right), \lambda}^{\left(\gamma_{r}\right),\left(l_{r}\right)}\left(z_{1}, \ldots, z_{r}\right) .
\end{aligned}
$$

\section{Singular integral equation}

In this section, we solve a singular integral equation with the generalized multivariable Mittag-Leffler function in the kernel. To do so, we first find the Laplace transform of the function $E_{\left(\rho_{r}\right), \lambda}^{\left(\gamma_{r}\right),\left(l_{r}\right)}\left((\mu x)^{\rho_{1}}, \ldots,(\mu x)^{\rho_{r}}\right)$ and we compute an integral involving the product of two generalized multivariable Mittag-Leffler functions.

We denote the Laplace transform of a function $f[16, \mathrm{p} .218]$ by

$$
\mathbb{L}[f(t)](p)=\tilde{f}(p)=\int_{0}^{\infty} \mathrm{e}^{-p t} f(t) d t \quad(\Re(p)>0) .
$$

Lemma 5.1 Let $p, \lambda, \mu, \rho_{j}, \gamma_{j}, l_{j} \in \mathbb{C}$ such that $\Re(p)>0 ; \Re(\mu)>0 ; \Re(\lambda)>0 ; \Re\left(\rho_{j}\right)>0 ; \Re\left(l_{j}\right)>$ $0(j=1, \ldots, r)$, we have

$$
\begin{aligned}
\mathbb{L} & {\left[t^{\lambda-1} E_{\left(\rho_{r}\right), \lambda}^{\left(\gamma_{r}\right),\left(l_{r}\right)}\left((\mu t)^{\rho_{1}}, \ldots,(\mu t)^{\rho_{r}}\right)\right](p) } \\
& =\frac{1}{p^{\lambda}} \sum_{k_{1}, \ldots, k_{r}=0}^{\infty} \frac{\left(\gamma_{1}\right)_{k_{1} l_{1}} \cdots\left(\gamma_{r}\right)_{k_{r} l_{r}}}{k_{1} ! \cdots k_{r} !}\left(\frac{\mu}{p}\right)^{\rho_{1} k_{1}+\cdots+\rho_{r} k_{r}} .
\end{aligned}
$$


Proof Using (5.1), we get

$$
\begin{aligned}
\mathbb{L} & {\left[t^{\lambda-1} E_{\left(\rho_{r}\right), \lambda}^{\left(\gamma_{r}\right),\left(l_{r}\right)}\left((\mu t)^{\rho_{1}}, \ldots,(\mu t)^{\rho_{r}}\right)\right](p) } \\
& =\sum_{k_{1}, \ldots, k_{r}=0}^{\infty} \frac{\left(\gamma_{1}\right)_{k_{1} l_{1}} \cdots\left(\gamma_{r}\right)_{k_{r} l_{r}}}{\Gamma\left(k_{1} \rho_{1}+\cdots+k_{r} \rho_{r}+\lambda\right)} \frac{\mu^{\rho_{1} k_{1}+\cdots+\rho_{r} k_{r}}}{k_{1} ! \cdots k_{r} !} \cdot \int_{0}^{\infty} \mathrm{e}^{-p t} t^{\rho_{1} k_{1}+\cdots+\rho_{r} k_{r}+\lambda-1} d t \\
& =\sum_{k_{1}, \ldots, k_{r}=0}^{\infty} \frac{\left(\gamma_{1}\right)_{k_{1} l_{1}} \cdots\left(\gamma_{r}\right)_{k_{r} l_{r}}}{\Gamma\left(k_{1} \rho_{1}+\cdots+k_{r} \rho_{r}+\lambda\right)} \frac{\mu^{\rho_{1} k_{1}+\cdots+\rho_{r} k_{r}}}{k_{1} ! \cdots k_{r} !} \cdot \frac{\Gamma\left(k_{1} \rho_{1}+\cdots+k_{r} \rho_{r}+\lambda\right)}{p^{k_{1} \rho_{1}+\cdots+k_{r} \rho_{r}+\lambda}} \\
& =\frac{1}{p^{\lambda}} \sum_{k_{1}, \ldots, k_{r}=0}^{\infty} \frac{\left(\gamma_{1}\right)_{k_{1} l_{1}} \cdots\left(\gamma_{r}\right)_{k_{r} l_{r}}}{k_{1} ! \cdots k_{r} !}\left(\frac{\mu}{p}\right)^{\rho_{1} k_{1}+\cdots+\rho_{r} k_{r}},
\end{aligned}
$$

where we used the well-known formula [16, p.218, Eq. (3)]

$$
\int_{0}^{\infty} \mathrm{e}^{-p t} t^{\lambda-1} d t=\frac{\Gamma(\lambda)}{p^{\lambda}} \quad(\min \{\Re(\lambda), \Re(p)\}>0) .
$$

Theorem 5.2 Let $p, \lambda, \mu, v, \rho_{j}, \gamma_{j}, l_{j}, \sigma_{j}, m_{j} \in \mathbb{C}$ such that $\Re(p)>0 ; \Re(\mu)>0 ; \Re(v)>0$; $\Re(\lambda)>0 ; \Re\left(\rho_{j}\right)>0 ; \Re\left(\sigma_{j}\right)>0 ; \Re\left(l_{j}\right)>0 ; \Re\left(m_{j}\right)>0(j=1, \ldots, r)$, we have

$$
\begin{aligned}
\int_{0}^{x}( & x-t)^{\lambda-1} E_{\left(\rho_{r}\right), \lambda}^{\left(\gamma_{r}\right),\left(l_{r}\right)}\left((\mu[x-t])^{\rho_{1}}, \ldots,(\mu[x-t])^{\rho_{r}}\right) \\
& \times t^{\nu-1} E_{\left(\rho_{r}\right), \nu}^{\left(\sigma_{r}\right),\left(m_{r}\right)}\left((\mu t)^{\rho_{1}}, \ldots,(\mu t)^{\rho_{r}}\right) d t \\
= & t^{\lambda+\nu-1} E_{\rho_{1}, \ldots, \rho_{r}, \rho_{1}, \ldots, \rho_{r}, \lambda+\nu}^{\gamma_{1}, \ldots, \gamma_{r}, \sigma_{1}, \ldots, \sigma_{1}, l_{1}, \ldots, l_{r}, m_{1}, \ldots, m_{r}}\left((\mu t)^{\rho_{1}}, \ldots,(\mu t)^{\rho_{r}},(\mu t)^{\rho_{1}}, \ldots,(\mu t)^{\rho_{r}}\right) .
\end{aligned}
$$

Proof With the help of the convolution theorem for the Laplace transform (see [17])

$$
\mathbb{L}\left[\int_{0}^{x} f(x-t) g(t) d t\right](p)=\mathbb{L}[f(x)](p) \mathbb{L}[g(x)](p),
$$

we have

$$
\begin{aligned}
\mathbb{L}\left[\int_{0}^{x}(x-t)^{\lambda-1} E_{\left(\rho_{r}\right), \lambda}^{\left(\gamma_{r}\right),\left(l_{r}\right)}\left((\mu[x-t])^{\rho_{1}}, \ldots,(\mu[x-t])^{\rho_{r}}\right)\right. \\
\left.\quad \times t^{\nu-1} E_{\left(\rho_{r}\right), v}^{\left(\sigma_{r}\right),\left(m_{r}\right)}\left((\mu t)^{\rho_{1}}, \ldots,(\mu t)^{\rho_{r}}\right) d t\right](p) \\
=\mathbb{L}\left[t^{\lambda-1} E_{\left(\rho_{r}\right), \lambda}^{\left(\gamma_{r}\right),\left(l_{r}\right)}\left((\mu t)^{\rho_{1}}, \ldots,(\mu t)^{\rho_{r}}\right)\right](p) \\
\quad \cdot \mathbb{L}\left[t^{\nu-1} E_{\left(\rho_{r}\right), \nu}^{\left(\sigma_{r}\right),\left(m_{r}\right)}\left((\mu t)^{\rho_{1}}, \ldots,(\mu t)^{\rho_{r}}\right)\right](p)
\end{aligned}
$$

From Lemma 5.1, we have

$$
\begin{gathered}
\mathbb{L}\left[\int_{0}^{x}(x-t)^{\lambda-1} E_{\left(\rho_{r}\right), \lambda}^{\left(\gamma_{r}\right),\left(l_{r}\right)}\left((\mu[x-t])^{\rho_{1}}, \ldots,(\mu[x-t])^{\rho_{r}}\right)\right. \\
\left.\quad \times t^{\nu-1} E_{\left(\rho_{r}\right), v}^{\left(\sigma_{r}\right),\left(m_{r}\right)}\left((\mu t)^{\rho_{1}}, \ldots,(\mu t)^{\rho_{r}}\right) d t\right](p)
\end{gathered}
$$




$$
\begin{aligned}
= & \frac{1}{p^{\lambda}} \sum_{k_{1}, \ldots, k_{r}=0}^{\infty} \frac{\left(\gamma_{1}\right)_{k_{1} l_{1}} \cdots\left(\gamma_{r}\right)_{k_{r} l_{r}}}{k_{1} ! \cdots k_{r} !}\left(\frac{\mu}{p}\right)^{\rho_{1} k_{1}+\cdots+\rho_{r} k_{r}} \\
& \cdot \frac{1}{p^{\mu}} \sum_{i_{1}, \ldots, i_{r}=0}^{\infty} \frac{\left(\sigma_{1}\right)_{i_{1} m_{1}} \cdots\left(\sigma_{r}\right)_{i_{r} m_{r}}}{i_{1} ! \cdots i_{r} !}\left(\frac{\mu}{p}\right)^{\rho_{1} i_{1}+\cdots+\rho_{r} i_{r}} \\
= & \frac{1}{p^{\lambda+v}} \sum_{k_{1}, \ldots, k_{r}, i_{1}, \ldots, i_{r}=0}^{\infty} \frac{\left(\gamma_{1}\right)_{k_{1} l_{1}} \cdots\left(\gamma_{r}\right)_{k_{r} l_{r}} \cdot\left(\sigma_{1}\right)_{i_{1} m_{1}} \cdots\left(\sigma_{r}\right)_{i_{r} m_{r}}}{k_{1} ! \cdots k_{r} ! i_{1} ! \cdots i_{r} !}\left(\frac{\mu}{p}\right)^{\rho_{1}\left(k_{1}+i_{1}\right)+\cdots+\rho_{r}\left(k_{r}+i_{r}\right)} \\
= & \mathbb{L}\left[t^{\lambda+v-1} E_{\rho_{1}, \ldots, \rho_{r}, \rho_{1}, \ldots, \rho_{r}, \lambda+\nu}^{\gamma_{1}, \gamma_{r}, \sigma_{1}, \ldots, \sigma_{1}, l_{1}, \ldots, l_{r}, m_{1}, \ldots, m_{r}}\left((\mu t)^{\rho_{1}}, \ldots,(\mu t)^{\rho_{r}},(\mu t)^{\rho_{1}}, \ldots,(\mu t)^{\rho_{r}}\right)\right](p)
\end{aligned}
$$

Finally, taking the inverse Laplace transform on both sides of (5.8), the result follows.

Now, let us consider the following convolution equation involving the generalized multivariable Mittag-Leffler in the kernel:

$$
\int_{0}^{x}(x-t)^{\lambda-1} E_{\left(\rho_{r}\right), \lambda}^{\left(\gamma_{r}\right),(1)}\left((\mu[x-t])^{\rho_{1}}, \ldots,(\mu[x-t])^{\rho_{r}}\right) \cdot \phi(t) d t=\psi(x)
$$

where $\Re(\alpha)>-1$.

Theorem 5.3 The singular integral equation (5.9) admits a locally integrable solution

$$
\phi(x)=\int_{0}^{x}(x-t)^{\omega-\lambda-1} E_{\left(\rho_{r}\right),(\omega-\lambda}^{\left(-\gamma_{r}\right),(1)}\left((\mu[x-t])^{\rho_{1}}, \ldots,(\mu[x-t])^{\rho_{r}}\right) \cdot{ }_{t} I_{0^{+}}^{-\omega} \psi(t) d t,
$$

provided that ${ }_{t} I_{0^{+}}^{-\omega} \psi(t)$ exists for $\Re(\omega)>\Re(\alpha+1)$ and is locally integrable for $0<t<\delta<\infty$.

Proof Applying the Laplace transform on both sides of (5.9), using the convolution theorem as well as Lemma 5.1, we find

$$
\frac{1}{p^{\lambda}} \sum_{k_{1}, \ldots, k_{r}=0}^{\infty} \frac{\left(\gamma_{1}\right)_{k_{1}} \cdots\left(\gamma_{r}\right)_{k_{r}}}{k_{1} ! \cdots k_{r} !}\left(\frac{\mu}{p}\right)^{\rho_{1} k_{1}+\cdots+\rho_{r} k_{r}} \cdot \mathbb{L}[\phi(t)](p)=\mathbb{L}[\psi(t)](p),
$$

which under the assumptions that $\left|\frac{\mu}{p}\right|<1$ can be rewritten as

$$
\frac{1}{p^{\lambda}} \prod_{j=1}^{r}\left(1-\left(\frac{\mu}{p}\right)^{\rho_{j}}\right)^{-\gamma_{j}} \cdot \mathbb{L}[\phi(t)](p)=\mathbb{L}[\psi(t)](p) .
$$

Therefore, we have

$$
\mathbb{L}[\phi(t)](p)=\left\{\prod_{j=1}^{r}\left(1-\left(\frac{\mu}{p}\right)^{\rho_{j}}\right)^{\gamma_{j}} p^{-\omega+\lambda}\right\} \cdot\left\{p^{\omega} \mathbb{L}[\psi(t)](p)\right\} .
$$

Taking the inverse Laplace transform on both sides of (5.13) and with the help of the following property [5, p.217, Eq. (3.8)]:

$$
p^{\mu} \mathbb{L}[f(t)](p)=\mathbb{L}\left[I_{0^{+}}^{-\mu} f(t)\right](p) \quad(\mu, p \in \mathbb{C} ; \Re(p)>0)
$$


which holds for suitable $f$, we thus obtain

$$
\phi(x)=\int_{0}^{x}(x-t)^{\omega-\lambda-1} E_{\left(\rho_{r}\right), \omega-\lambda}^{\left(-\gamma_{r}\right),(1)}\left((\mu[x-t])^{\rho_{1}}, \ldots,(\mu[x-t])^{\rho_{r}}\right) \cdot{ }_{t} I_{0^{+}}^{-\omega} \psi(t) d t .
$$

\section{Competing interests}

The authors declare that they have no competing interests.

\section{Authors' contributions}

All authors completed the paper together. All authors read and approved the final manuscript.

\section{Author details}

'Department of Mathematics and Computer Science, University of Quebec at Chicoutimi, Quebec, G7H 2B1, Canada.

2Eastern Mediterranean University, Gazimagusa, TRNC, Mersin, 10, Turkey.

Received: 10 July 2014 Accepted: 3 September 2014 Published: 24 Sep 2014

\section{References}

1. Mitag-Leffler, GM: Sur la nouvelle function $E_{\alpha}(x)$. C. R. Acad. Sci. Paris 137, 554-558 (1903)

2. Mitag-Leffler, GM: Sur la représentation analytique d'une fonction monogène (cinquième note). Acta Math. 29, 101-181 (1905)

3. Saxena, RK, Mathai, AM, Haubold, HJ: On fractional kinetic equations. Astrophys. Space Sci. 282(1), $281-287$ (2002)

4. Wiman, A: Über den fundamental Satz in der Theorien der Funktionen $E_{\alpha}(x)$. Acta Math. 29, 191-201 (1905)

5. Prabhakar, TR: A singular integral equation with a generalized Mittag-Leffler function in the kernel. Yokohama Math. J. 19, 7-15 (1971)

6. Srivastava, HM, Tomovski, Z: Fractional calculus with an integral operator containing a generalized Mittag-Leffler function in the kernel. Appl. Math. Comput. 211(1), 198-210 (2009)

7. Saxena, RK, Kalla, SL, Saxena, R: Multivariable analogue of generalized Mittag-Leffler function. Integral Transforms Spec. Funct. 22, 533-548 (2011)

8. Srivastava, HM, Daoust, MC: Certain generalized Neumann expansion associated with Kampé de Fériet function. Proc. K. Ned. Akad. Wet., Ser. A, Indag. Math. 31, 449-457 (1969)

9. Srivastava, HM, Karlsson, PW: Multiple Gaussian Hypergeometric Series. Ellis Horwood, Chichester (1985)

10. Özarslan, MA: On a singular integral equation including a set of multivariate polynomials suggested by Laguerre polynomials. Appl. Math. Comput. 229, 350-358 (2014)

11. Erdélyi, A: Beitrag zur theorie der konfluenten hypergeometrischen funktionen von mehreren veränderlichen. Sitzungsber. Akad. Wiss. Wien, Math.-Naturw. Kl., Abt. Ila 146, 431-467 (1937)

12. Konhauser, JDE: Biorthogonal polynomials suggested by the Laguerre polynomials. Pac. J. Math. 21, 303-314 (1967)

13. Erdélyi, A, Magnus, W, Oberhettinger, F, Tricomi, F: Higher Transcendental Functions, vols. 1-3. McGraw-Hill, New York (1953)

14. Kilbas, AA, Srivastava, HM, Trujillo, JJ: Theory and Applications of Fractional Differential Equations. North-Holland Mathematical Studies, vol. 204. Elsevier, Amsterdam (2006)

15. Jaimini, BB, Gupta, J: On certain fractional differential equations involving generalized multivariable Mittag-Leffle function. Note Mat. 32(2), 141-156 (2012)

16. Srivastava, HM, Manocha, HL: A Treatise on Generating Functions. Ellis Horwood, Chichester (1984)

17. Titchmarsh, EC: Introduction to the Theory of Fourier Integrals, 3rd edn. Chelsea, New York (1986). The first edition in Oxford University Press, Oxford (1937)

10.1186/1687-1847-2014-252

Cite this article as: Gaboury and Özarslan: Singular integral equation involving a multivariable analog of

Mittag-Leffler function. Advances in Difference Equations 2014, 2014:252 